\title{
Expression of tung tree diacylglycerol acyltransferase 1 in E. coli
}

\author{
Heping Cao ${ }^{*}$, Dorselyn C Chapital, Jay M Shockey and K Thomas Klasson
}

\begin{abstract}
Background: Diacylglycerol acyltransferases (DGATs) catalyze the final and rate-limiting step of triacylglycerol (TAG) biosynthesis in eukaryotic organisms. Database search has identified at least 59 DGAT1 sequences from 48 organisms, but the expression of any DGAT1 as a full-length protein in E. coli had not been reported because DGAT1s are integral membrane proteins and difficult to express and purify. The objective of this study was to establish a procedure for expressing full-length DGAT1 in E. coli.

Results: An expression plasmid containing the open reading frame for tung tree (Vernicia fordii) DGAT1 fused to maltose binding protein and poly-histidine affinity tags was constructed and expressed in E. coli BL21(DE3). Immunoblotting showed that the recombinant DGAT1 (rDGAT1) was expressed, but mostly targeted to the membranes and insoluble fractions. Extensive degradation also occurred. Nonetheless, the fusion protein was partially purified from the soluble fraction by Ni-NTA and amylose resin affinity chromatography. Multiple proteins co-purified with DGAT1 fusion protein. These fractions appeared yellow in color and contained fatty acids. The rDGAT1 was solubilized from the insoluble fraction by seven detergents and urea, with SDS and Triton X-100 being the most effective detergents. The solubilized rDGAT1 was partially purified by Ni-NTA affinity chromatography. PreScission protease digestion confirmed the identity of rDGAT1 and showed extensive precipitation following $\mathrm{Ni}$ NTA affinity purification.

Conclusions: This study reports the first procedure for expressing full-length DGAT1 from any species using a bacterial expression system. The results suggest that recombinant DGAT1 is degraded extensively from the carboxyl terminus and associated with other proteins, lipids, and membranes.
\end{abstract}

\section{Background}

The functions of a considerable percentage of the proteins encoded by the sequenced genomes of many organisms including human, mouse, Arabidopsis, and rice are not clear. The immediate task of post-genomic biology is to determine the biological functions of proteins coded for by these genes. Many endogenous proteins occur in extremely low abundance but recombinant protein can be used as alternative sources to endogenous proteins for studying protein structure and function [1], for making high-titer antibodies [2-4], and for producing pharmaceutical reagents. However, a great number of proteins are difficult to express due to protein insolubility, protein degradation, and low-level

\footnotetext{
* Correspondence: Heping.Cao@ars.usda.gov

Commodity Utilization Research Unit, Southern Regional Research Center, Agricultural Research Service, U.S. Department of Agriculture, 1100 Robert E. Lee Blvd., New Orleans, Louisiana 70124, USA
}

protein expression [5]. Therefore, production of high quality recombinant protein requires optimization of protein expression and purification procedures.

Diacylglycerol acyltransferases (DGATs) are responsible for the last and rate-limiting step of triacylglycerol (TAG) biosynthesis in eukaryotic organisms. DGAT genes have been isolated from many organisms [6]. At least two forms of DGATs are present in mammals $[7,8]$ and plants $[9,10]$ with additional forms reported in burning bush (Euonymus alatus) [11] and peanut [12]. DGAT isoforms have nonredundant functions in TAG biosynthesis in plants such as tung tree (Vernicia fordii) [10] and animals such as mice [13]. Tung tree genome has two well-known DGAT isoforms (DGAT1 and DGAT2) which are targeted to distinct subdomains of the ER membrane, and likely carry out different roles in seed lipid metabolism [10]. Mice deficient in DGAT1 are viable, have modest decreases in TAG, and are

\section{Biomed Central}

(c) 2011 Cao et al; licensee BioMed Central Ltd. This is an Open Access article distributed under the terms of the Creative Commons Attribution License (http://creativecommons.org/licenses/by/2.0), which permits unrestricted use, distribution, and reproduction in any medium, provided the original work is properly cited. 
resistant to diet-induced obesity $[14,15]$. Mice deficient in DGAT2 have severe reduction of TAG and die shortly after birth [13]. The fact that DGAT1 is unable to compensate for the deficiency in DGAT2 knockout mice indicates that each DGAT isoform has unique functions in TAG biosynthesis during mammal development.

Database search has identified at least 59 DGAT1 sequences from 48 organisms (Figure 1). However, there is a gap of knowledge between the gene sequences and the proteins coded for by the DGAT genes [6]. Although DGAT expression was reported in several studies, only limited success has been achieved due to the facts that DGATs are integral membrane proteins $[10,16]$ and are difficult to express and purify using heterologous hosts $[17,18]$. Information regarding the expression of DGAT1 gene in E. coli is limited. The amino terminal 116 amino acid residues of oilseed rape (Brassica napus) DGAT1 was expressed as a His-tag protein in E. coli [18]. Similar studies reported on the production of the amino terminal 95 residues of mouse DGAT1 in E. coli [19]. However, the expression of DGAT1 as a full-length protein in E. coli had not been reported. The objective of this study was to develop a reliable procedure for the expression of a full-length DGAT1 in E. coli using tung tree DGAT1 (one of the two isoforms from tung tree) as the model protein.

\section{Results}

\section{Construction of bacterial expression plasmid}

Plasmid pMBP-DGAT1-His was engineered to express the full-length Vernicia fordii (tung tree) DGAT1 in an $E$. coli protein expression system. The recombinant protein MBP-DGAT1-His (rDGAT1) contained a MBP (maltose binding protein) at the amino terminus and 6 histidine residues (His) at the carboxyl terminus (Figure 2). A PreScission protease cleavage site was engineered between the MBP and DGAT1 fusion partners. The "Methods" section describes the details of the plasmid construction.

\section{Degradation of recombinant DGAT1 in the soluble fraction of $E$. coli}

The expression of rDGAT1 was induced by IPTG in $E$. coli strain BL21(DE3) and detected by immunoblotting using anti-MBP-hTTP and anti-MBP-mTTP polyclonal antibodies, which were raised in rabbits against purified recombinant human and mouse TTP proteins fused to MBP $[2,3]$. These antibodies have been shown to react with MBP and MBP fusion proteins with high specificity $[2,3]$.

Anti-MBP-mTTP antibodies detected a number of bands ranging from approximately $40 \mathrm{kDa}$ (corresponding to that of MBP, $42 \mathrm{kDa}$ ) to $60 \mathrm{kDa}$ and a trace

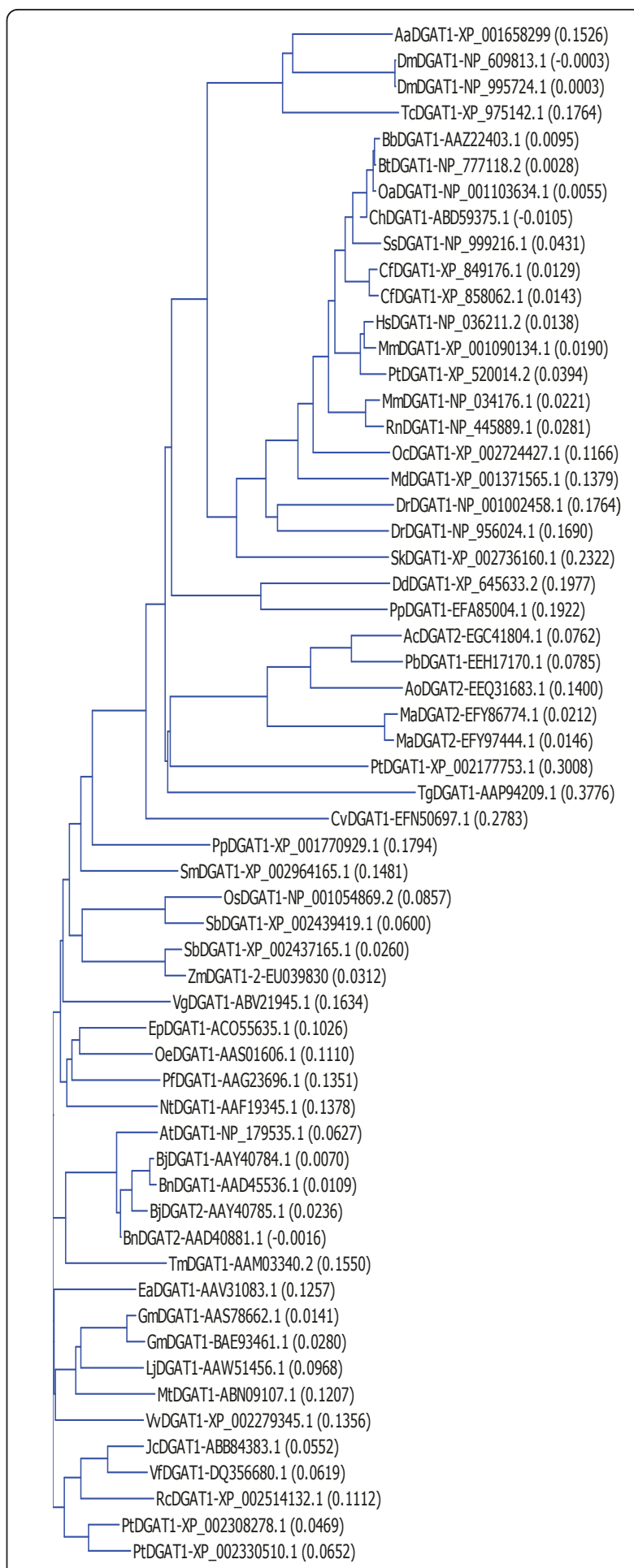

Figure 1 Phylogenetic analysis of DGAT1s. The evolutionary relationships among the 59 DGAT1s from 48 organisms were analyzed by phylogenetic analysis based on the Neighbor-Joining method of Saitou and Nei [35]. The name of each protein sequence consists of the initials of the organism followed by the assigned subfamily of DGATs in the databases and the GenBank accession number. The numbers in the parenthesis following DGAT names are the calculated distance values, which reflect the degree of 
divergence between all pairs of DGAT sequences analyzed. The abbreviations of the organisms are: Ac, Ajellomyces capsulatus; Ao, Arthroderma otae; At, Arabidopsis thaliana; Bb, Bubalus bubalis; $\mathrm{Bj}$, Brassica juncea; Bn, Brassica napus; Bt, Bos taurus; Cf, Canis familiaris: Cv, Chlorella variabilis; Dd, Dictyostelium discoideum; Dm, Drosophila melanogaster; Dr, Danio rerio; Ea, Euonymus alatus; Ep, Echium pitardii; Gm, Glycine max; Hs, Homo sapiens; Jc, Jatropha curcas; Lj, Lotus japonicas; Ma in MaDGAT1-EFY86774.1, Metarhizium acridum; Ma in MaDGAT1-EFY97444.1, Metarhizium anisopliae; Md, Monodelphis domestica; Mm in MmDGAT1-XP_001090134.1, Macaca mulatta; Mm in MmDGAT1-NP_034176.1, Mus musculus; Mt, Medicago truncatula; Nt, Nicotiana tabacum; Oa, Ovis aries; Oc, Oryctolagus cuniculus; Oe, Olea europaea; Os, Oryza sativa; Pb, Paracoccidioides brasiliensis; Pf, Perilla frutescens; Pp in PpDGAT1EFA85004.1, Polysphondylium pallidum; Pp in PpDGAT1XP_001770929.1, Physcomitrella patens; Pt in PtDGAT1-XP_520014.2, Pan troglodytes; Pt in PtDGAT1a-XP_002308278.1, Populus trichocarpa; Rc, Ricinus communis; Rn, Rattus norvegicus; Sb, Sorghum bicolor; Sk, Saccoglossus kowalevskii; Sm, Selaginella moellendorffii; Ss, Sus scrofa; Tc, Tribolium castaneum; Tg, Toxoplasma gondii; Tm, Tropaeolum majus; Vf, Vernicia fordii; Vg, Vernonia galamensis; Vv, Vitis vinifera; Zm, Zea mays.

amount of protein corresponding to the full-size rDGAT1 (909 amino acid residues, $102 \mathrm{kDa}$ ) (Figure $3 \mathrm{~A})$. There was no significant difference in protein expression levels among different starting colonies of the bacterium. The expression levels, judged from the overall full-length and degradation products, were decreased under longer expression time tested (Figure 3A). Less but similar sizes of the full-length and

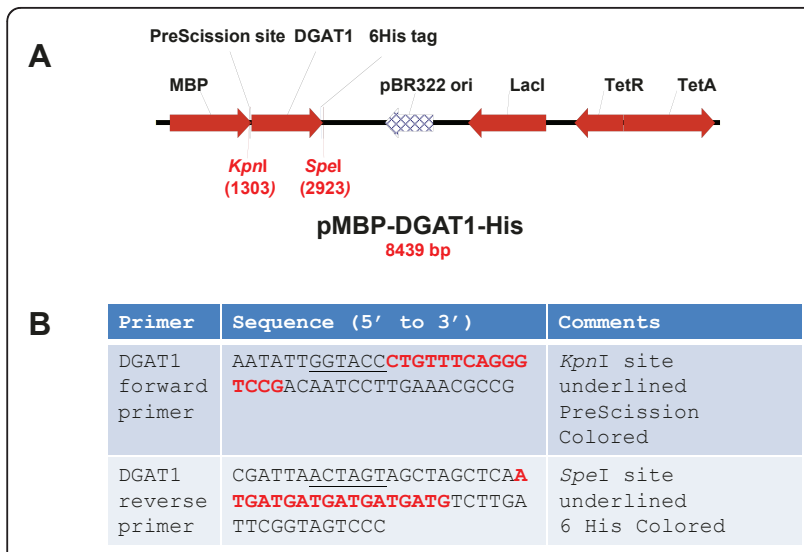

Figure 2 Plasmid map used for the construction of an E. coli expression vector and primer sequences used for PCRamplification of the DGAT1 insert. (A) Diagram of the expression plasmid pMBP-DGAT1-His. Plasmid pMBP-hTTP [1] was used to express full-length DGAT1 in E. coli. Tung DGAT1 DNA was subcloned as described in the Materials and Methods section. (B) Primers for construction of the $E$. coli expression plasmid. The sequences for restriction enzyme digestion sites are underlined. DGAT1 forward primer and reverse primer have sequences coding for a Prescission protease digestion site and 6 histidine residues, respectively. degradation products were detected in the cells without IPTG induction (Figure 3A), indicating a high basal level expression of the recombinant protein under uninduced conditions. The $60 \mathrm{kDa}$ band corresponds to protein smaller than the $80-\mathrm{kDa}$-sized MBP-mTTP fusion protein (a positive control for the antibodies in lane 2 of Figure 3A). Recombinant DGAT1 expression was not significantly different when protein expression was induced at $25^{\circ} \mathrm{C}$ or $37^{\circ} \mathrm{C}$, nor under culture medium with or without $0.2 \%$ glucose (data not shown). Similar results were obtained using anti-MBP-hTTP antibodies and the commercial anti-MBP antibodies (data not shown). In agreement with the immunoblotting results shown in Figure 3A, a protein staining gel showed that no distinct band with a molecular mass corresponding to the full-length rDGAT1 was observed in the $10,000 \mathrm{~g}$ supernatant from the uninduced and IPTG-induced cells (Figure 3B).

As a control experiment, plasmid pMAL-c2X vector was transformed into the same type of E. coli. Like rDGAT1 expression in the uninduced cells described above, MBP protein was clearly produced in the uninduced cells (Figure $3 \mathrm{C}$ ). In contrast to the minimal expression of rDGAT1 in E. coli (Figure 3A and 3B), MBP was massively induced by IPTG in the same type of cells (Figure 3C), which was specifically recognized by the commercial anti-MBP antibodies [1] and the same anti-MBP-hTTP antibodies [2] and anti-MBP-mTTP antibodies [3] used in the current study. These results suggest that rDGAT1 was expressed well but was extensively degraded in $E$. coli under the experimental conditions.

\section{Localization of recombinant DGAT1 in the insoluble fraction and membranes of $E$. coli}

Amylose resin affinity chromatography was used to identify the trace amount of the full-length protein as rDGAT1 shown in Figure 3. Based on the expression studies described above, DGAT1 fusion protein expression in E. coli was scaled up. The $10,000 g$ supernatant was applied onto an MBPTrap HP column. After extensive washing, the bound proteins were eluted with 20 $\mathrm{mM}$ maltose. However, no clear protein peak was eluted with maltose as judged from UV absorbance at $280 \mathrm{~nm}$ (data not shown). Immunoblotting confirmed that the great majority if not all of the immuno-reactive proteins did not bind to amylose resin and was enriched in the unbound fractions (Figure 4A).

To investigate if the recombinant protein was in the insoluble fraction, the $10,000 \mathrm{~g}$ pellet was sonicated extensively in the same homogenization buffer. This supernatant was used for MBPTrap column purification. The great majority of the "released" recombinant DGAT1 did not bind to the column (as in the case with 


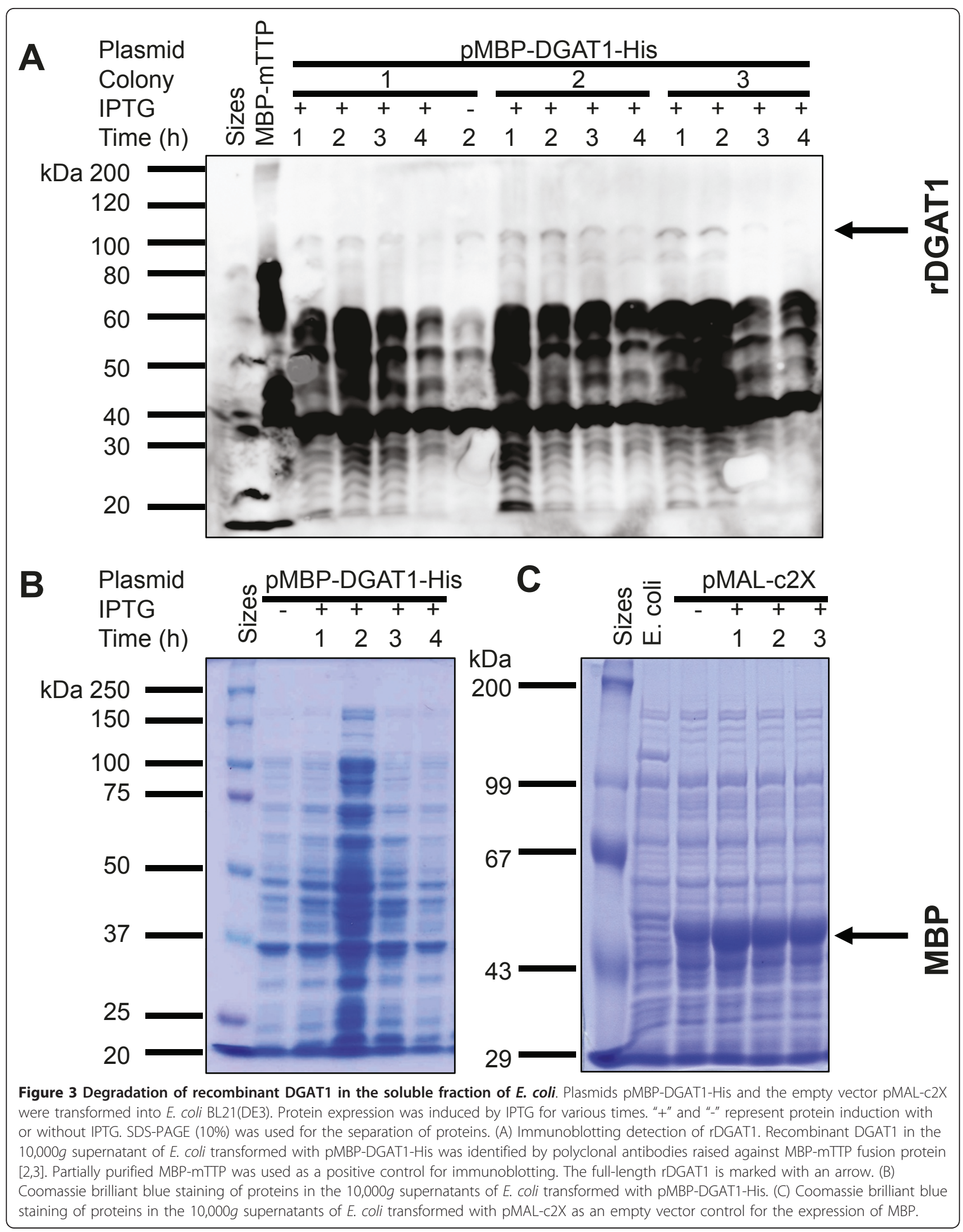




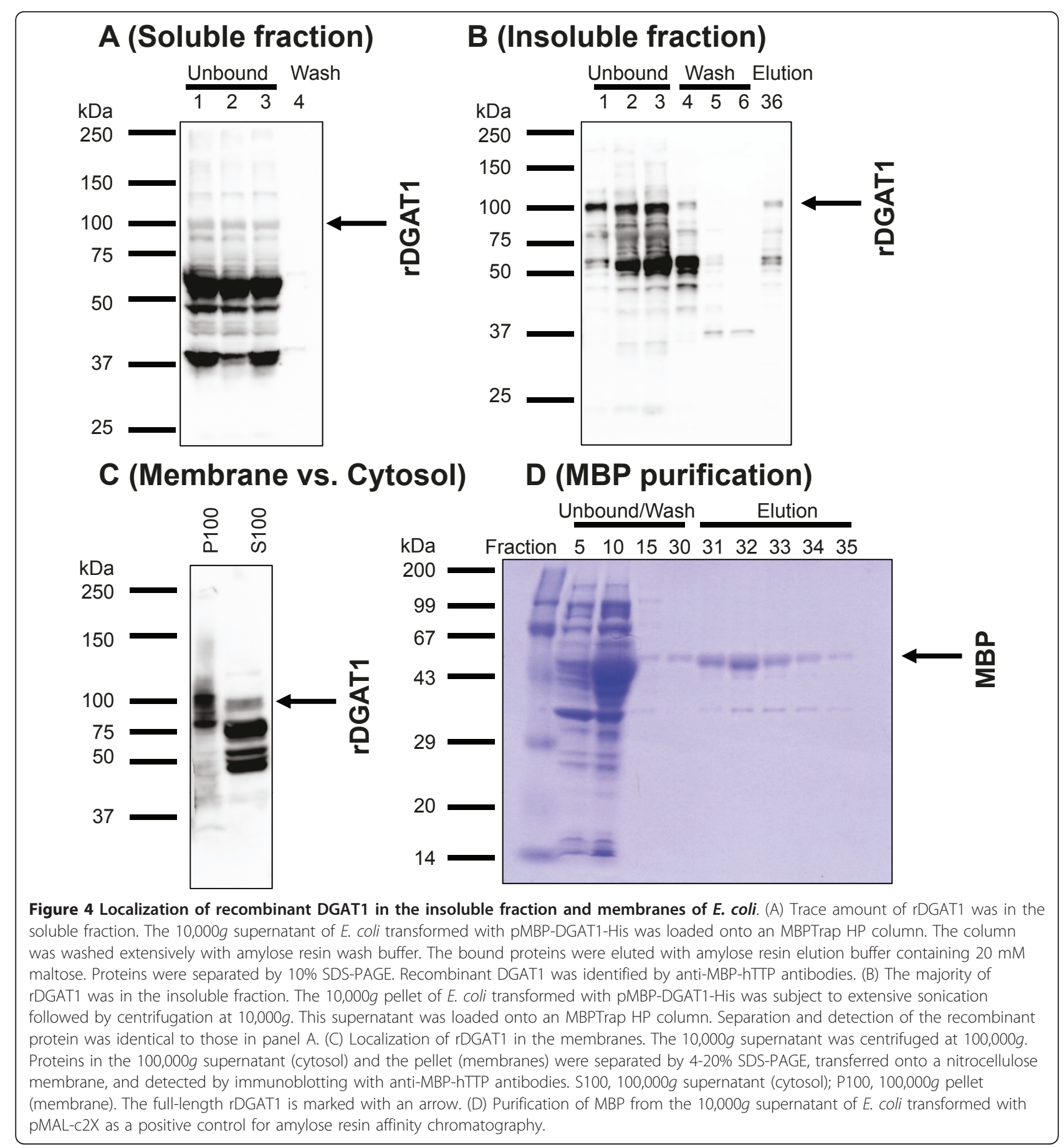

the soluble rDGAT1) and was mainly recovered in the unbound fractions (Figure 4B). However, immunoblotting showed that the full-length rDGAT1 was much more enriched in the pellet than in the soluble fraction of $E$. coli and some full-length protein was eluted from the column (Figure $4 \mathrm{~A}$ versus $4 \mathrm{~B}$, right lanes).

To localize the trace amount of the recombinant protein in the supernatant shown in panel $\mathrm{A}$, the $10,000 \mathrm{~g}$ supernatant was centrifuged at $100,000 \mathrm{~g}$. Immunoblotting showed that rDGAT1 was mainly associated with the membrane fraction and only a smaller fraction of rDGAT1 in the cytosol (Figure 4C).

Figure $4 \mathrm{~A}$ and $4 \mathrm{~B}$ showed that rDGAT1 bound to amylose resin poorly because little rDGAT1 was recovered in the eluted fractions. To provide a positive control for amylose resin affinity purification, E. coli extract 
with overexpressed MBP shown in Figure 3C was applied to amylose resin. Significant amounts of MBP bound to the affinity resin and was purified to near homogeneity by the affinity chromatography, although lots of the recombinant MBP did not bind to the same affinity beads (Figure 4D). These results suggest that rDGAT1 may be folded in a way to prevent MBP fusion partner from binding to the affinity resin.

\section{Purification of recombinant DGAT1 with Ni-NTA affinity chromatography}

$\mathrm{Ni}$-NTA beads were used to test the purification of the recombinant protein using the $10,000 \mathrm{~g}$ supernatant. The bound proteins were eluted with successively increasing imidazole concentrations ranging from 50 to $1000 \mathrm{mM}$. SDS-PAGE showed that the purified fractions contained a number of proteins as shown by Coomassie blue staining (Figure 5A). The majority of the bound recombinant protein (as detected by immunoblotting) was eluted with 200-250 mM imidazole in the elution buffer and no recombinant protein was detected in the washes (Figure 5B). rDGAT1 only partially bound to Ni-NTA beads because a significant amount of the full-length rDGAT1 was recovered in the unbound fraction (data not shown). As expected, MBP and most of the degradation products were not bound to the Ni-NTA beads because the immunoreactive bands on the blot corresponded to the full-length rDGAT1 (Figures 3, 4 vs. 5B). A protein band with approximately twice the size $(>150$ $\mathrm{kDa}$ ) of the full-length rDGAT1 was seen in the eluted fractions on the immunoblot after SDS-denatured gel separation (Figure 5B). A similar immunoreactive band

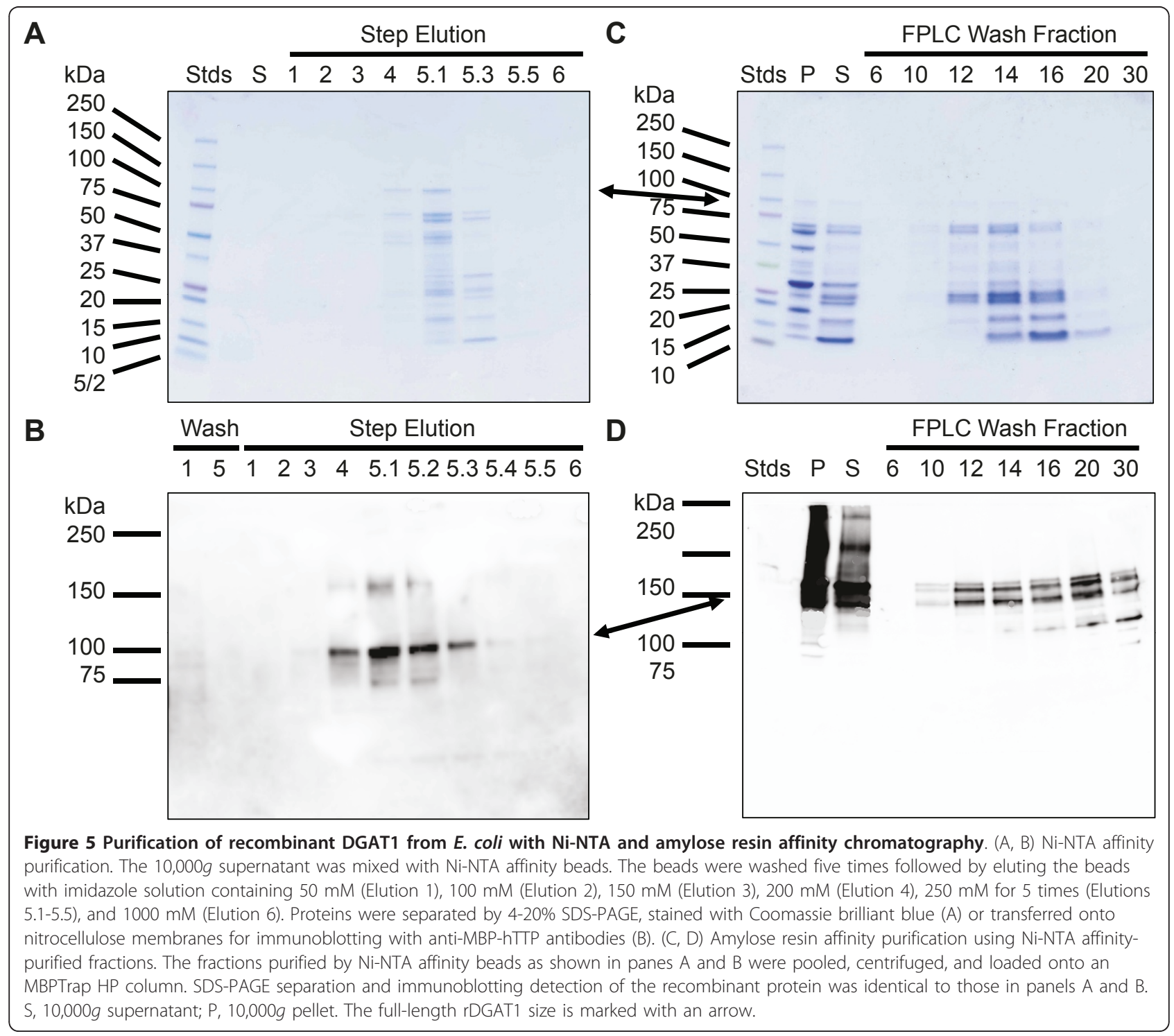


with the higher molecular mass was also observed on other immunoblots (see below).

\section{Purification of recombinant DGAT1 with tandem Ni-NTA and amylose resin affinity chromatography}

The proteins eluted by $250 \mathrm{mM}$ imidazole from $\mathrm{Ni}$ NTA affinity beads (Figure 5A) were pooled and centrifuged at $10,000 \mathrm{~g}$. The supernatant was loaded onto an amylose resin affinity column. FPLC chromatogram showed that the great majority of proteins were washed off the column and little protein was bound to the MBPTrap column (data not shown), similar to those observed using the $10,000 \mathrm{~g}$ supernatant (Figure 5A). Coomassie blue staining showed that the additional affinity purification step did not improve the purity (Figure $5 \mathrm{C})$. Immunoblotting showed that part of the recombinant protein was precipitated (Figure $5 \mathrm{D}$, lane $\mathrm{P}$ vs. line S) and rDGAT1 was detected in the unbound fractions (Figure 5D) but undetectable in the eluted fractions (data not shown).

SDS solubilization and purification of recombinant DGAT1 from insoluble fraction

Immunoblotting showed that the great majority of rDGAT1 was recovered in the 10,000 $g$ pellet (Figure 4B).
Therefore, SDS was used to solubilize rDGAT1 from $10,000 \mathrm{~g}$ as well as the $25,000 \mathrm{~g}$ pellet fractions followed by purification with Ni-NTA affinity chromatography. Immunoblotting showed that full-length rDGAT1 was barely detectable in the 25,000 g supernatant and the wash (Figure 6A). Some full-length rDGAT1 and several degraded protein bands were detected in the unbound fraction and the major protein band was detected in the eluted fraction (Figure 6A). The full-length rDGAT1 was stable in cells under IPTG induction for 2-24 h as demonstrated in the purified fractions from the SDSsolubilized pellet (Figure 6B).

\section{Detergent and urea solubilization and purification of recombinant DGAT1 from insoluble fraction}

As the results indicated that rDGAT1 was associated with the insoluble pellet, an attempt was made to solubilize the recombinant protein from the $10,000 \mathrm{~g}$ pellet with seven different detergents (Brij 35, CHAPS, NP-40, SDS, Triton X-100, Tween 20 and Tween 80) and urea followed by purification with Ni-NTA affinity chromatography. Immunoblotting showed that SDS was the most effective detergent for rDGAT1 solubilization, while only small amounts of the full-length rDGAT1 was detected in the solubilized fractions by other detergents

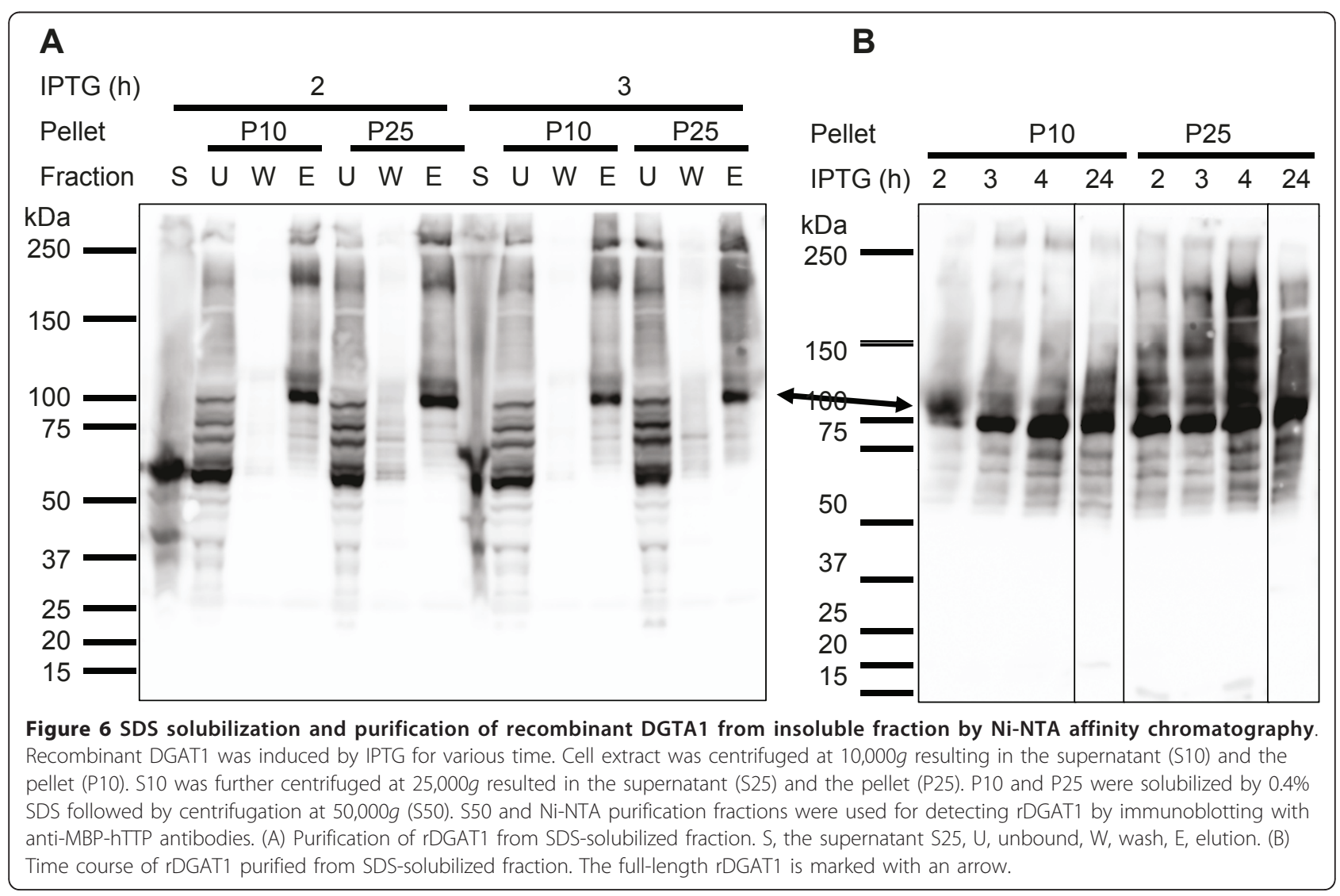


(Figure 7A, lanes 2-7). The optimal concentrations for SDS solubilization were $0.3-1 \%$ (Figure 7A, lanes 11-13). Ni-NTA affinity purification showed that solubilization by both SDS and Triton X-100 resulted in the highest yields of full-length rDGAT1 (Figure 7B, lanes 5-6 and 10-13). Therefore, various concentrations of Triton X100 were tested for the solubilization of rDGAT1 from the pellet. Immunoblotting showed that $0.3-1 \%$ Triton $\mathrm{X}-100$ were the optimal concentrations for the extraction (Figure 7C, lanes 2-8) and purification (Figure 7D, lanes 2-8). Urea at $4 \mathrm{M}$ and $6 \mathrm{M}$ also solubilized the fusion protein to a significant level (Figure 7C, lanes 912). However, Ni-NTA affinity chromatography indicated that rDGAT1 from urea-solubilized samples bound to the affinity beads poorly because much less of the full-length protein was obtained in the urea-solubilized protein solution (Figure 7D, lanes 9-12). Silver staining of the purified fractions indicated multiple proteins co-purified with the recombinant protein (data not shown). Extensive efforts were directed to purify rDGAT1 from the Ni-NTA purified proteins following SDS and Triton X100 solubilization. However, the protein could not be purified to near homogeneity by amylose resin affinity, Superose 12 size exclusion, and Mono $\mathrm{Q}$ anion exchange chromatography (data not shown).

\section{PreScission protease digestion of recombinant DGAT1}

Immunoblotting results showed that MBP-TTP antibodies detected multiple protein bands from soluble fraction (Figure 3), insoluble fraction (Figure 4B), membrane fraction (Figure 4C), Ni-NTA and amylose resin affinity-purified fractions (Figure 5), and detergentand urea-solubilized fractions (Figures 6 and 7). To confirm the identity of the full-length rDGAT1, PreScission protease digestion was performed using rDGAT1 purified by Ni-NTA affinity chromatography (Figure 5A)

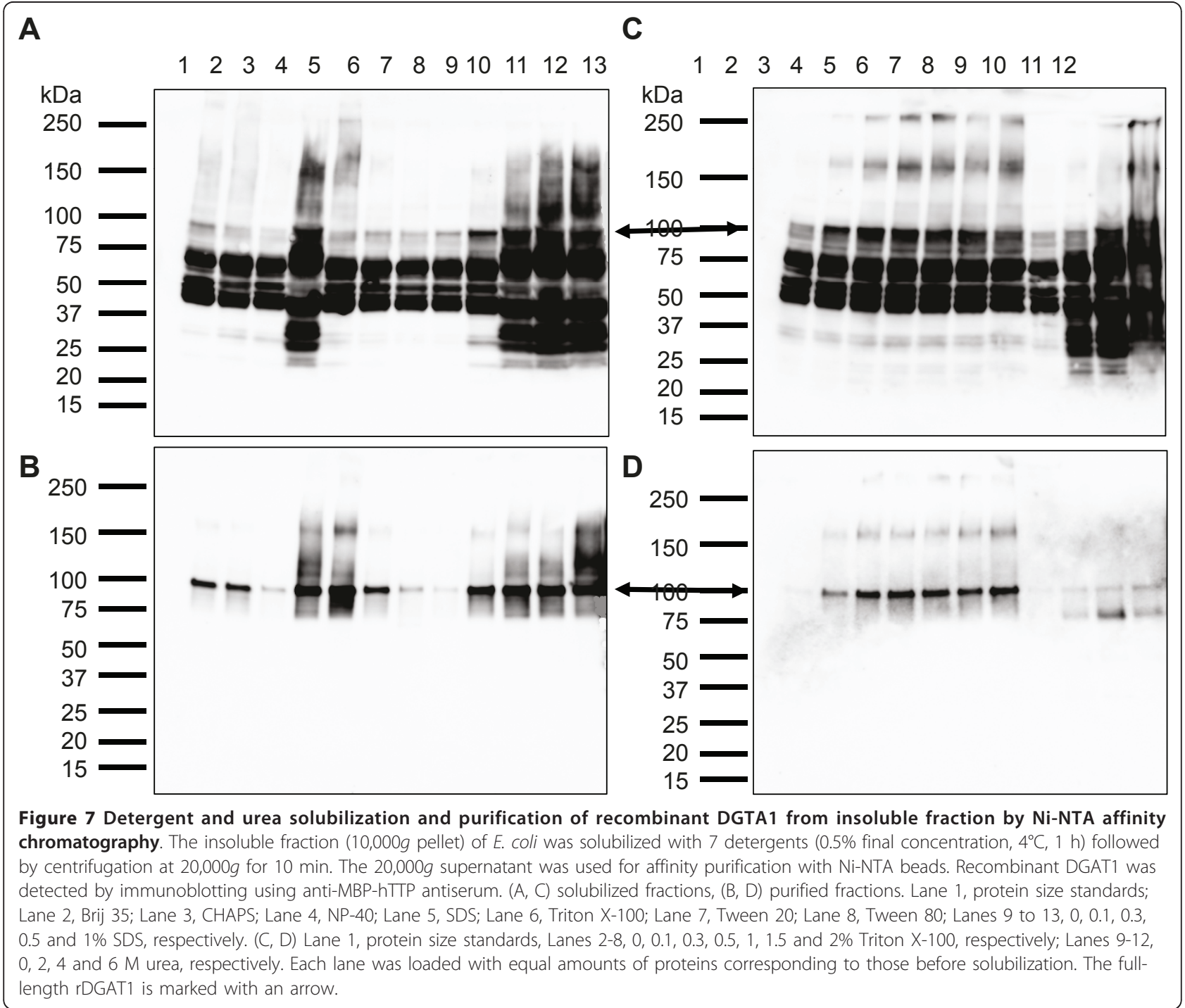


because a PreScission protease cleavage site was engineered between MBP and DGAT1 fusion partners (Figure 2). The rDGAT1 sample was digested by the protease overnight followed by centrifugation at $10,000 \mathrm{~g}$. Immunoblotting showed that a protein band with the size of MBP was detected in the soluble fraction but not in the undigested protein sample (Figure 8, lane 1 vs. lane 2). It also showed that the great majority of rDGAT1 was recovered in the pellet following digestion (Figure 8, lane 2 vs. lane 3 ). These results confirmed the identity of rDGAT1 and showed extensive precipitation of rDGAT1 following purification.

\section{Discussion}

Diacylglycerol acyltransferase families (DGATs) catalyze the final and rate-limiting step of triacylglycerol (TAG) biosynthesis in eukaryotic organisms. Understanding the roles of DGATs will help to create transgenic plants with value-added properties and provide information for therapeutic intervention for obesity and related diseases. Database search has identified at least 59 DGAT1 sequences from 48 organisms (Figure 1), but the expression of any DGAT1 as a full-length protein in E. coli

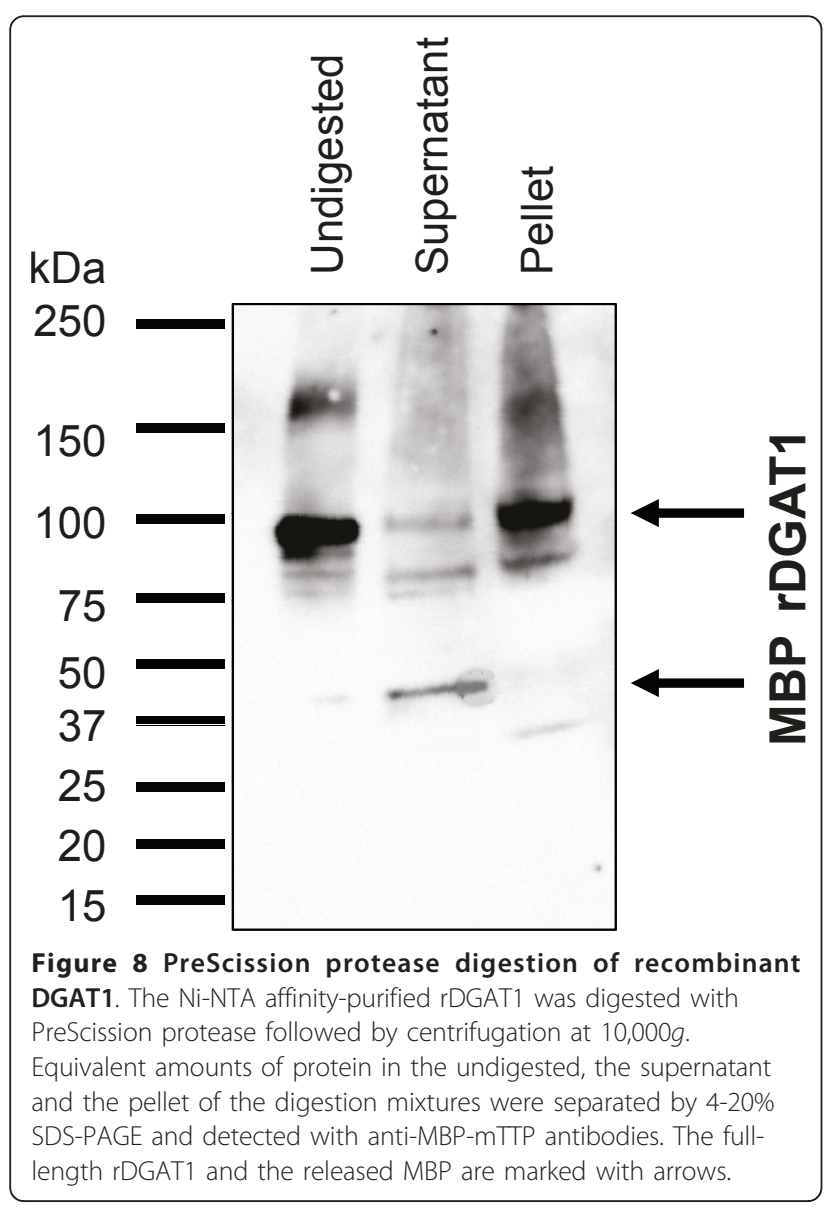

had not been reported. Here a procedure was described for expressing recombinant full-length DGAT1 in a bacterial expression system. The hydrophilic amino termini of DGAT1 from mouse and oilseed rape had previously been expressed as His-tagged fusion proteins in $E$. coli $[18,19]$. However, the full-length DGAT1 was not expressed in $E$. coli previously because it is an integral membrane protein with 8 putative membrane-spinning domains using topology modeling [18-20], although mice DGAT1 has only three transmembrane domains using protease protections assays and indirect immunofluorescence in conjunction with selective permeabilization of cellular membranes [21]. In this study, the fulllength DGAT1 was fused to MBP at the amino terminus and His-tag at the carboxyl terminus of the protein. The full-length DGAT1 was successfully expressed in $E$. coli and it is possible that the fusion to MBP contributed to the results, as MBP has been shown to increase the solubility of target proteins such as human and mouse TTP $[1,4,22]$.

Purification of recombinant DGATs from any source represents a challenge. Only a few studies in E. coli have been directly related to the purification of recombinant DGATs [6]. Expression of soluble peanut DGAT (DGAT3, $42 \mathrm{kDa}$ ) in $E$. coli resulted in high levels of DGAT activity and the formation of labeled TAG [12]. The recombinant $\mathrm{N}$-terminal region of Brassica napus DGAT1 (BnDGAT1 $\left.1_{(1-116)} \mathrm{His}_{6}\right)$ was purified from E. coli with a predicted molecular mass of 13,278 Da, which was confirmed by MALDI-TOF mass spectrometry; however, the apparent molecular mass on SDS-PAGE was doubled and the native size was four times of the size of the monomer due to self-association. Full-length DGAT1 was not successfully expressed in E. coli $[18,20]$. In the study herein, we engineered double affinity tags for facilitating purification of recombinant DGAT1 from E. coli. Recombinant DGAT1 was only partially purified from the extract by either type of affinity beads or both kinds of beads, probably due to extensive precipitation (Figure 8). Preliminary attempts to measure the activity of rDGAT1 using cold oleoyl-CoA and sn-1,2-diolein did not result in visible TGA spot on TLC plates (data not shown), probably due to the low sensitivity of cold substrate. Further experiments are required to demonstrate the biological activity of rDGAT1 using radioactive oleoyl-CoA. Our current data, together with the various published reports cited here, underline the tremendous challenges that exist for the purification of recombinant full-length DGAT proteins.

Several lines of evidence support the assignment of the full-length rDGAT1 on various immunoblots despite the facts that MBP-TTP antibodies detected multiple protein bands from soluble fraction, insoluble fraction, membrane fraction, Ni-NTA and amylose resin affinity- 
purified fractions, and detergent- and urea-solubilized fractions. First, MBP-TTP antibodies have been wellcharacterized in a number of previous publications which cross-react specifically with both MBP and TTP [2,3,23-25]. Second, the detection of MBP by anti-MBPTTP antibodies $[2,3]$ is identical to the commercial antiMBP antibodies [1]. Third, the size of the full-length rDGAT1 on immunoblots corresponded to the calculated size of the protein. Fourth, affinity purification with Ni-NTA beads based on the His-tag at the carboxyl terminus of rDGAT1 resulted in the full-length rDGAT1 being the most predominant band on the immunoblots (Figures 5B, 6, 7B and 7D). These results also suggest that the multiple lower molecular mass bands on immunoblots are degradation products from the carboxyl terminus of rDGAT1 because they were detected in amylose resin affinity-purified protein samples (Figure 4) but not if any in Ni-NTA affinity-purified protein samples (Figures 5B, 6, 7B and 7D). Finally, the full-length rDGAT1 was partially digested by PreScission protease resulting in the release of MBP in the soluble fraction (Figure 8).

During the purification process, we observed several lines of evidence that suggest rDGAT1 is directly or indirectly associated with other proteins, lipids, and plasma membranes. First, rDGAT1 is associated with $E$. coli plasma membranes, because the majority of DGAT1 fusion protein was detected in the $100,000 \mathrm{~g}$ plasma membranes instead of the 100,000 $\mathrm{g}$ cytosol (Figure 4C). Second, the partially purified proteins eluted from affinity beads contained multiple proteins even after extensive washes (Figure 5A and 5C). Finally, the purified protein samples appeared yellow in color. Gas chromatography confirmed that Ni-NTA purified rDGAT1 fraction contained fatty acids (data not shown). These observations are in agreement with several previous reports showing that DGAT is associated with lipids or lipid bodies [9,26-28], lipid droplets of cultured mouse adipocytes (equivalent to oil bodies in plant seeds) [29] and ER membranes [10]. As a positive control for the expression and purification experiments, the MBP fusion partner was massively induced by IPTG in the same type of $E$. coli and purified from the soluble fraction to near homogeneity by a single step of amylose resin affinity chromatography. Therefore, the direct or indirect association of rDGAT1 with other proteins, lipids, and plasma membranes may explain why tandem affinity beads were not effective in the purification of rDGAT1 to homogeneity.

\section{Conclusions}

The current study reports the first procedure for expressing the full-length recombinant DGAT1 from any species using a bacterial expression system. The results suggest that recombinant DGAT1 is extensively degraded from the carboxyl terminus and is directly or indirectly associated with other proteins, lipids, and membranes. Although the protein was not purified to homogeneity in the current study, the ability to express full-length DGAT1 in E. coli could provide the basis for future purification of the protein.

\section{Methods}

\section{Bacterial expression plasmid construction}

The plasmid pMBP-DGAT1-His was engineered to express the full-length tung tree (Vernicia fordii) type 1 diacylglycerol acyltransferase (DGAT1, GenBank Accession No. DQ356680[10]) in E. coli protein expression system. The recombinant protein MBP-DGAT1-His (rDGAT1) contained MBP (maltose binding protein) at the amino terminus and 6 histidine residues (His) at the carboxyl terminus. Plasmid pMBP-hTTP, the cloning vector, was reported previously [1]. Plasmid pMBPDGAT1-His was constructed by replacing the hTTP fragment in plasmid pMBP-hTTP with the PCR-amplified DGAT1 fragment at the KpnI and SpeI sites (Figure 2A). A previously constructed DGAT1 plasmid was used as the template for PCR amplification of the DGAT1 DNA open reading frame [10]. The DGAT1 forward primer contained a $K p n \mathrm{I} / A s p 718 \mathrm{I}$ restriction enzyme recognition site followed by a PreScission protease cleavage site (5'-CTGTTTCAGGGTCCG-3') [1] which codes for 5 amino acid residues (LFQGP) between MBP and DGAT1 protein sequences (Figure 2B). DGAT1 reverse primer contained sequence coding for a His-tag (5'-ATGATGATGATGATGATG-3') at the carboxyl terminus of the recombinant protein (Figure $2 \mathrm{~B}$ ). The plasmid construction was confirmed by restriction enzyme digestion and DNA sequencing using the GenomeLab Dye Terminator Cycle Sequencing-Quick Start Kit and CEQ 8000 Genetic Analysis System (Beckman Coulter). The commercial plasmid pMAL-c2X (New England BioLabs) was used as an empty vector control for the expression and purification of MBP fusion partner.

\section{Expression of recombinant DGAT1 in E. coli}

Plasmid pMBP-DGAT1-His was transformed into the $E$. coli BL21(DE3) strain by electroporation. The optimum conditions for rDGAT1 expression were as follows: three separate single colonies were inoculated into Luria-Bertani (LB)-tetracycline $(15 \mu \mathrm{g} / \mathrm{mL})$ medium (LBTet) and grown overnight with shaking at $37^{\circ} \mathrm{C}$. The overnight culture was inoculated at a 1:20 dilution into fresh medium and grown for about $4 \mathrm{~h}$ at $37^{\circ} \mathrm{C}$ to reach an optical cell density of approximately $0.6-1.0$ at $\mathrm{OD}_{600}$ $\mathrm{nm}$. Isopropylthio- $\beta$-D-galactoside (IPTG) was added to the culture medium $(0.5 \mathrm{mM}$ final concentration) and protein expression was induced at $25^{\circ} \mathrm{C}$ for up to $4 \mathrm{~h}$, or 
in control experiments they were left uninduced for $2 \mathrm{~h}$. Cells were collected by centrifugation at $5,000 \mathrm{~g}$ for 10 min and homogenized by sonication in homogenization buffer (3-4 mL/g wet cells) containing amylose resin wash buffer $(20 \mathrm{mM}$ Tris- $\mathrm{HCl}, \mathrm{pH} 7.4,200 \mathrm{mM} \mathrm{NaCl}$, $10 \mathrm{mM} \beta$-mercaptoethanol, $1 \mathrm{mM}$ EDTA) or nickelnitrilotriacetic agarose (Ni-NTA agarose from Qiagen) resin wash buffer $\left(50 \mathrm{mM} \mathrm{NaH}{ }_{2} \mathrm{PO}_{4}, \mathrm{pH} 7.4,300 \mathrm{mM}\right.$ $\mathrm{NaCl}, 10 \mathrm{mM} \beta$-mercaptoethanol, 0.05\% Tween-20), plus $0.2-1 \mathrm{mM}$ phenylmethylsulfonyl fluoride (PMSF), and 1:100-1:500 dilution of protease inhibitor cocktail (Sigma, cat \#P8340). The homogenate was centrifuged at $2,000 \mathrm{~g}$ for $10 \mathrm{~min}$ to remove cell debris and the resulting supernatant was centrifuged at $10,000 \mathrm{~g}$ for 10 min to remove inclusion bodies and protein aggregates $[30,31]$. The supernatant and the pellet were evaluated for the expression levels and solubility of rDGAT1.

\section{Purification of recombinant DGAT1 with Ni-NTA affinity chromatography}

The rDGAT1 was partially purified from E. coli by batch method from the $10,000 \mathrm{~g}$ supernatant using Ni-NTA beads according to similar procedures [2]. The 10,000 g supernatant was mixed with Ni-NTA Agarose (Qiagen). The mixtures were incubated at $4^{\circ} \mathrm{C}$ with rotation for 3 $\mathrm{h}$ followed by centrifugation at $1,000 \mathrm{~g}$ for $5 \mathrm{~min}$. The beads were washed five times each with 5 bead-volume of Ni-NTA resin wash buffer. The bound proteins were eluted from the beads by gravity flow in a Bio-Rad minicolumn with increasing concentrations of imidazole (Sigma) in Ni-NTA wash buffer.

\section{Purification of recombinant DGAT1 with amylose resin affinity chromatography}

The rDGAT1 was subjected to purification with an amylose resin affinity column (MBPTrap HP column, GE Healthcare Life Sciences) using fast protein liquid chromatography (FPLC) (GE Healthcare Life Sciences). This system had previously been used for purification of MBPTTP and MBP-ZFP36L1 fusion proteins $[1,2,4]$. The $10,000 \mathrm{~g}$ supernatant or the partially purified rDGAT1 from Ni-NTA batch purification was loaded onto the column. The column was washed with 5 bed-volume of amylose resin wash buffer and then eluted with 10 bedvolume of amylose resin elution buffer $(20 \mathrm{mM}$ maltose in amylose resin wash buffer) followed by $0.5 \mathrm{M} \mathrm{NaOH}$ wash of the column. MBP was also affinity-purified from E. coli transformed with plasmid pMAL-c2x (New England Biolabs) by amylose resin affinity chromatography and used as a control in the experiments [2].

\section{Localization of recombinant DGAT1 in E. coli}

$E$. coli cells were homogenized by sonication as described above. The homogenate was sequentially centrifuged at 2,000 $\mathrm{g}$ for $10 \mathrm{~min}$ and $10,000 \mathrm{~g}$ for 20 min. The $10,000 \mathrm{~g}$ supernatant $(2 \mathrm{~mL})$ was subjected to $100,000 \mathrm{~g}$ centrifugation to generate the cytosol and plasma membrane pellet [32]. The 100,000 $g$ pellets were suspended in $0.2 \mathrm{~mL}$ membrane suspension buffer containing $10 \mathrm{mM}$ Tris, $\mathrm{pH} 7.4,1 \mathrm{mM}$ EDTA, and $0.2 \mathrm{M}$ sucrose. Anti-MBP-hTTP antibodies were used to identify rDGAT1 in various fractions.

\section{Purification of recombinant DGAT1 from detergent- solubilized $E$. coli pellets}

Cellular fractionation studies indicated that rDGAT1 could be recovered from the $10,000 \mathrm{~g}$ and $25,000 \mathrm{~g}$ pellet. Therefore, the pellets were suspended in Ni-NTA sonication buffer and used for detergent solubilization at $4^{\circ}$ $\mathrm{C}$ for $1 \mathrm{~h}$ with $0.5 \%$ of detergents: Brij 35 (Aldrich Chemical Corp.), CHAPS (Sigma), NP-40 (Sigma), SDS (Fisher), Triton X-100 (International Biotechnologies Inc.), Tween 20 and Tween 80 (Sigma). The pellets were further solubilized at $4^{\circ} \mathrm{C}$ for $1 \mathrm{~h}$ by various concentrations of SDS (0.1, 0.3, 0.5, and 1\%), Triton X-100 (0.1$2 \%)$, and urea $(2-6 \mathrm{M})$. The solubilization mixtures were centrifuged at 20,000-50,000 $\mathrm{g}$ for $10 \mathrm{~min}$. The supernatant was used for rDGAT1 detection and purification with Ni-NTA affinity chromatography as described above.

\section{PreScission protease digestion of recombinant DGAT1}

PreScission protease digestion was performed to confirm the identity of the full-length rDGAT1 according to a previous protocol [1]. Briefly, rDGAT1 purified by NiNTA affinity chromatography (Figure 5A) was mixed with PreScission protease (GE Healthcare Life Sciences). The digestion mixture was incubated at room temperature overnight with shaking followed by centrifugation at $10,000 \mathrm{~g}$. Equivalent amounts of protein in the undigested, the supernatant and pellet of the digestion mixtures were separated with 4-20\% SDS-PAGE and detected with anti-MBP-mTTP antibodies.

\section{Protein determination, SDS-PAGE, and immunoblotting}

Protein concentrations were determined with the Bradford method using the Protein Assay Dye Reagent Concentrate (Bio-Rad Laboratories) following 0.5 M NaOH treatment of the protein samples [33]. Proteins were separated by SDS-PAGE $(10 \%, 15 \%$, or $4-20 \%)$ and visualized by staining with Coomassie brilliant blue (Sigma) or silver staining reagent (Bio-Rad Laboratories) [2]. DGAT1 fusion protein was detected by immunoblotting following previously described procedures using nitrocellulose membranes and SuperSignal West Pico Chemiluminescent Substrate (Pierce) [2,33]. The primary antibodies were rabbit anti-MBP-hTTP antibodies [2] and anti-MBP-mTTP antibodies [3], as well as the 
commercial anti-MBP antibodies (New England BioLabs). The secondary antibodies were affinity-purified goat anti-rabbit IgG $(\mathrm{H}+\mathrm{L})$ horseradish peroxidase conjugate (GAR-HRP) with human IgG absorbed (Bio-Rad Laboratory).

\section{Lists of abbreviations}

DGAT: diacylglycerol acyltransferase; His: poly histidine; IPTG: isopropylthio- $\beta$-D-galactoside; MBP: maltose binding protein; Ni-NTA: nickel-nitrilotriacetic agarose; PAGE: polyacrylamide gel electrophoresis; rDGAT1: recombinant diacylglycerol acyltransferase; TAG: triacylglycerol; TTP: tristetraprolin.

\section{Acknowledgements and Funding}

The authors thank Drs. John M. Dyer (USDA-ARS-Arid-Land Agricultural Research Center), Thomas J. Caperna (USDA-ARS-Beltsville Agricultural Research Center), Jeffrey W. Cary and Sophie M. Uchimiya (USDA-ARSSouthern Regional Research Center) for helpful comments on the manuscript and Mr. O.D. Howard Jr. and Dr. Stephanie A. Boone for technical assistance. A preliminary report was presented at the Experimental Biology 2011 in Washington DC on April 9- 13, 2011 [34]. This work was supported by USDA-ARS Quality and Utilization of Agricultural Products Research Program.

\section{Authors' contributions}

$\mathrm{HC}$ conceived, designed, and performed the experiment and wrote the manuscript. DCC performed some cell culture and immunoblotting analyses. JMS and KTK are involved in providing intellectual insights and revising the manuscript. All authors read and approved the manuscript.

Received: 15 April 2011 Accepted: 11 July 2011 Published: 11 July 2011

\section{References}

1. Cao H, Dzineku F, Blackshear PJ: Expression and purification of recombinant tristetraprolin that can bind to tumor necrosis factor-alpha mRNA and serve as a substrate for mitogen-activated protein kinases. Arch Biochem Biophys 2003, 412:106-120.

2. Cao H: Expression, purification, and biochemical characterization of the antiinflammatory tristetraprolin: a zinc-dependent mRNA binding protein affected by posttranslational modifications. Biochemistry 2004, 43:13724-13738.

3. Cao H, Tuttle JS, Blackshear PJ: Immunological characterization of tristetraprolin as a low abundance, inducible, stable cytosolic protein. $J$ Biol Chem 2004, 279:21489-21499.

4. Cao H, Lin R, Ghosh S, Anderson RA, Urban JF Jr: Production and characterization of ZFP36L1 antiserum against recombinant protein from Escherichia coli. Biotechnol Prog 2008, 24:326-333.

5. Cao H: Recombinant protein production technology. I Jiangxi Agric Univ 2010, 32:1018-1031.

6. Cao H: Bioengineering recombinant diacylglycerol acyltransferases. In Bioengineering. Edited by: Carpi A. Rijeka, Croatia: InTech; 2011:1-16.

7. Cases S, Smith SJ, Zheng YW, Myers HM, Lear SR, Sande E, Novak S, Collins C, Welch CB, Lusis AJ, Erickson SK, Farese RV Jr: Identification of a gene encoding an acyl CoA:diacylglycerol acyltransferase, a key enzyme in triacylglycerol synthesis. Proc Natl Acad Sci USA 1998, 95:13018-13023.

8. Cases $S$, Stone SJ, Zhou P, Yen E, Tow B, Lardizabal KD, Voelker T, Farese RV $\mathrm{Jr}$ : Cloning of DGAT2, a second mammalian diacylglycerol acyltransferase, and related family members. J Biol Chem 2001, 276:38870-38876.

9. Lardizabal KD, Mai JT, Wagner NW, Wyrick A, Voelker T, Hawkins DJ: DGAT2 is a new diacylglycerol acyltransferase gene family: purification, cloning, and expression in insect cells of two polypeptides from Mortierella ramanniana with diacylglycerol acyltransferase activity. J Biol Chem 2001, 276:38862-38869.
10. Shockey JM, Gidda SK, Chapital DC, Kuan JC, Dhanoa PK, Bland JM, Rothstein SJ, Mullen RT, Dyer JM: Tung tree DGAT1 and DGAT2 have nonredundant functions in triacylglycerol biosynthesis and are localized to different subdomains of the endoplasmic reticulum. Plant Cell 2006, 18:2294-2313.

11. Durrett TP, McClosky DD, Tumaney AW, Elzinga DA, Ohlrogge J, Pollard M A distinct DGAT with sn-3 acetyltransferase activity that synthesizes unusual, reduced-viscosity oils in Euonymus and transgenic seeds. Proc Natl Acad Sci USA 2010, 107:9464-9469.

12. Saha S, Enugutti B, Rajakumari S, Rajasekharan R: Cytosolic triacylglycerol biosynthetic pathway in oilseeds. Molecular cloning and expression of peanut cytosolic diacylglycerol acyltransferase. Plant Physiol 2006, 141:1533-1543.

13. Stone SJ, Myers HM, Watkins SM, Brown BE, Feingold KR, Elias PM, Farese RV Jr: Lipopenia and skin barrier abnormalities in DGAT2-deficient mice. J Biol Chem 2004, 279:11767-11776.

14. Chen HC, Smith SJ, Ladha Z, Jensen DR, Ferreira LD, Pulawa LK, McGuire JG, Pitas RE, Eckel RH, Farese RV Jr: Increased insulin and leptin sensitivity in mice lacking acyl CoA:diacylglycerol acyltransferase 1. J Clin Invest 2002, 109:1049-1055.

15. Smith SJ, Cases S, Jensen DR, Chen HC, Sande E, Tow B, Sanan DA, Raber J, Eckel RH, Farese RV Jr: Obesity resistance and multiple mechanisms of triglyceride synthesis in mice lacking Dgat. Nat Genet 2000, 25:87-90.

16. Stone SJ, Levin MC, Farese RV Jr: Membrane topology and identification of key functional amino acid residues of murine acyl-CoA:diacylglycerol acyltransferase-2. J Biol Chem 2006, 281:40273-40282.

17. Cheng D, Meegalla RL, He B, Cromley DA, Billheimer JT, Young PR: Human acyl-CoA:diacylglycerol acyltransferase is a tetrameric protein. Biochem J 2001, 359:707-714

18. Weselake RJ, Madhavji M, Szarka SJ, Patterson NA, Wiehler WB, Nykiforuk CL, Burton TL, Boora PS, Mosimann SC, Foroud NA, Thibault BJ, Moloney MM, Laroche A, Furukawa-Stoffer TL: Acyl-CoA-binding and self-associating properties of a recombinant $13.3 \mathrm{kDa} \mathrm{N}$-terminal fragment of diacylglycerol acyltransferase-1 from oilseed rape. BMC Biochem 2006, 7:24.

19. Siloto RM, Madhavji M, Wiehler WB, Burton TL, Boora PS, Laroche A, Weselake RJ: An N-terminal fragment of mouse DGAT1 binds different acyl-CoAs with varying affinity. Biochem Biophys Res Commun 2008, 373:350-354

20. Hobbs DH, Lu C, Hills MJ: Cloning of a cDNA encoding diacylglycerol acyltransferase from Arabidopsis thaliana and its functional expression. FEBS Lett 1999, 452:145-149.

21. McFie PJ, Stone SL, Banman SL, Stone SJ: Topological orientation of acylCoA:diacylglycerol acyltransferase-1 (DGAT1) and identification of a putative active site histidine and the role of the $\mathrm{n}$ terminus in dimer/ tetramer formation. J Biol Chem 2010, 285:37377-37387.

22. Kapust RB, Waugh DS: Escherichia coli maltose-binding protein is uncommonly effective at promoting the solubility of polypeptides to which it is fused. Protein Sci 1999, 8:1668-1674.

23. Cao H, Deterding $L$, Venable JD, Kennington EA, Yates JR, Tomer KB, Blackshear PJ: Identification of the anti-inflammatory protein tristetraprolin as a hyperphosphorylated protein by mass spectrometry and site-directed mutagenesis. Biochem J 2006, 394:285-297.

24. Cao H, Lin R: Phosphorylation of recombinant tristetraprolin in vitro. Protein J 2008, 27:163-169.

25. Cao H, Lin R: Quantitative evaluation of His-tag purification and immunoprecipitation of tristetraprolin and its mutant proteins from transfected human cells. Biotechnol Prog 2009, 25:461-467.

26. Bouvier-Nave P, Benveniste P, Oelkers P, Sturley SL, Schaller H: Expression in yeast and tobacco of plant CDNAs encoding acyl CoA:diacylglycerol acyltransferase. Eur J Biochem 2000, 267:85-96.

27. Christiansen K: Triacylglycerol synthesis in lipid particles from baker's yeast (Saccharomyces cerevisiae). Biochim Biophys Acta 1978, 530:78-90.

28. Kamisaka Y, Mishra S, Nakahara T: Purification and characterization of diacylglycerol acyltransferase from the lipid body fraction of an oleaginous fungus. J Biochem 1997, 121:1107-1114.

29. Kuerschner $L$, Moessinger $C$, Thiele C: Imaging of lipid biosynthesis: how a neutral lipid enters lipid droplets. Traffic 2008, 9:338-352.

30. Ren $\mathrm{Q}$, De RG, Kessler B, Witholt B: Recovery of active medium-chainlength-poly-3-hydroxyalkanoate polymerase from inactive inclusion bodies using ion-exchange resin. Biochem J 2000, 349:599-604. 
31. Massie HR, Zimm BH: Molecular weight of the DNA in the chromosomes of E. coli and B. subtilis. Proc Natl Acad Sci USA 1965, 54:1636-1641.

32. Romanov GA, Spichal L, Lomin SN, Strnad M, Schmulling T: A live cell hormone-binding assay on transgenic bacteria expressing a eukaryotic receptor protein. Anal Biochem 2005, 347:129-134.

33. Cao H, Sullivan TD, Boyer CD, Shannon JC: Bt1, a structural gene for the major 39-44 kDa amyloplast membrane polypeptides. Physiol Plant 1995, 95:176-186.

34. Cao H, Chapital DC, Howard OD, Jiang XN, Shockey JM, Klasson KT: Purification of recombinant tung tree diacylglycerol acyltransferases from E. coli. FASEB J 2011, 25:765-8.

35. Saitou N, Nei M: The neighbor-joining method: a new method for reconstructing phylogenetic trees. Mol Biol Evol 1987, 4:406-425.

doi:10.1186/1472-6750-11-73

Cite this article as: Cao et al:: Expression of tung tree diacylglycerol acyltransferase 1 in E. coli. BMC Biotechnology 2011 11:73.

Submit your next manuscript to BioMed Central and take full advantage of:

- Convenient online submission

- Thorough peer review

- No space constraints or color figure charges

- Immediate publication on acceptance

- Inclusion in PubMed, CAS, Scopus and Google Scholar

- Research which is freely available for redistribution

Submit your manuscript at www.biomedcentral.com/submit 